\title{
Novel regulation of Ras proteins by direct tyrosine phosphorylation and dephosphorylation
}

\author{
László Buday ${ }^{1,2} \cdot$ Virág Vas ${ }^{1}$ \\ Received: 7 June 2020 / Accepted: 19 June 2020 / Published online: 16 September 2020 \\ (C) The Author(s) 2020
}

\begin{abstract}
Somatic mutations in the RAS genes are frequent in human tumors, especially in pancreatic, colorectal, and non-small-cell lung cancers. Such mutations generally decrease the ability of Ras to hydrolyze GTP, maintaining the protein in a constitutively active GTP-bound form that drives uncontrolled cell proliferation. Efforts to develop drugs that target Ras oncoproteins have been unsuccessful. Recent emerging data suggest that Ras regulation is more complex than the scientific community has believed for decades. In this review, we summarize advances in the "textbook" view of Ras activation. We also discuss a novel type of Ras regulation that involves direct phosphorylation and dephosphorylation of Ras tyrosine residues. The discovery that pharmacological inhibition of the tyrosine phosphoprotein phosphatase SHP2 maintains mutant Ras in an inactive state suggests that SHP2 could be a novel drug target for the treatment of Ras-driven human cancers.
\end{abstract}

Keywords Ras $\cdot$ Ras signaling $\cdot$ SOS $\cdot$ Tyrosine phosphorylation $\cdot$ SHP2 $\cdot$ Cancer therapy

\section{Introduction}

The products of the $R A S$ family of proto-oncogenes are lowmolecular-weight guanine nucleotide-binding proteins that mediate cell growth, survival, and differentiation via interactions with a variety of effector proteins $[1,2]$. Ras proteins are enzymes capable of hydrolyzing bound GTP to GDP and inorganic phosphate. Cycling between GDP-bound inactive and GTP-bound active forms is facilitated by guanine nucleotide exchange factors (GEF) and GTPase-activating proteins (GAPs) via a mechanism that is common in the Ras superfamily $[3,4]$. The three human $R A S$ genes encode four highly similar proteins: H-Ras, N-Ras, and K-Ras4A and K-Ras4B. The expression of two protein products from the mammalian $K-R A S$ gene results from the use of alternative fourth exons.

Single-base substitutions in codons 12,13 , or 61 of $R A S$ are among the most frequent oncogenic mutations in human cancers [5]. These mutations activate Ras by eliminating its GTP

László Buday

buday.laszlo@ttk.hu

1 Institute of Enzymology, Research Centre for Natural Sciences, Budapest 1117, Hungary

2 Department of Medical Chemistry, Semmelweis University Medical School, Budapest 1094, Hungary hydrolysis activity. Despite the high degree of similarity between the isoforms, K-Ras is the most frequently mutated; indeed, K-Ras mutations have been identified in $22 \%$ of all tumors investigated (compared with $8 \%$ for N-Ras and $3 \%$ for H-Ras) [6, 7].

\subsection{Conventional regulation of Ras activation}

The first conceptualization of Ras activation was established in the early 1990s. According to this early model, growth factors, e.g., epidermal growth factor (EGF), induce a rapid dimerization and autophosphorylation of their receptors at the plasma membrane. Phosphotyrosine residues in the noncatalytic region of the receptors bind a variety of signaling molecules possessing SH2 or PTB domains, including the Grb2 adaptor protein. Grb2 is a small ubiquitously expressed and highly conserved protein with a central $\mathrm{SH} 2$ domain flanked by two SH3 domains. Binding of Grb2 to activated receptors via its $\mathrm{SH} 2$ domain recruits the SOS-Grb2 complex from the cytosol, placing SOS in proximity to the plasma membrane where it can stimulate the exchange of GDP for GTP on membrane-bound Ras. These early studies suggested that SOS translocation to the plasma membrane was sufficient for Ras activation [8-10].

However, the importance of Grb2-mediated membrane recruitment of SOS was challenged by several critical questions emerging from subsequent studies. For example, a 
transforming mutant of SOS1 that was unable to bind Grb2 seemed to mediate normal downstream signaling [11]. Another study suggested that Grb2 negatively modulates SOS activity under basal conditions [12]. In addition, SOS constructs lacking the Grb2-binding proline-rich regions were found to be successfully recruited to Ras-enriched membranes [13]. How can one explain SOS activation at the plasma membrane if Grb2's activity is limited at the membrane? The solution may come from the multidomain structure of SOS, which affords it multiple functions, i.e., catalysis, membrane binding (protein-protein and proteinlipid interactions), allosteric regulation, and autoinhibition. SOS possesses a histone-fold domain, Dbl homology (DH) and Pleckstrin homology (PH) domains, a Ras exchanger motif (REM), the catalytic cell division cycle 25 (Cdc25) domain, and a proline-rich $\mathrm{C}$-terminal region that binds Grb2 [10]. Two of the abovementioned domains exert autoinhibitory effects on SOS. The proline-rich region of SOS binds the SH3 domains of Grb2, facilitating SOS recruitment to the plasma membrane. On the other hand, this interaction overcomes negative regulation of SOS by its own C-terminus. Similarly, contact between the SOS PH domain and negatively charged phospholipids also induces conformational changes that allow binding of allosteric Ras to the REM domain, which results in processive catalysis of nucleotide exchange on the substrate Ras in the Cdc25 domain [14-16] (Fig. 1).

Biologically active Ras is strictly localized to membranes determined by specific lipid modifications [17]; therefore, it is not surprising that SOS, upon activation in tyrosine signaling pathway, also binds to membranes containing allosteric and substrate Ras. Furthermore, it has also been shown that constitutive membrane localization of SOS activates Ras [18]. A recent series of elegant experiments from the laboratory of Jay T. Groves shed new light on the dynamics of SOS activation. Via single-molecule assays, they identified distinct activation states of SOS, reflecting different stable configurations of the protein complex itself [19]. More interestingly, they recognized a subgroup of SOS molecules in which Ras GTP bound to the SOS allosteric site had a smaller activating effect compared with that of bound Ras-GDP [19]. It seems well-established that SOS translocation to the plasma membrane requires a number of protein-lipid and protein-protein interactions, as described above; however, it has long remained unclear how SOS activity is downregulated in tyrosine kinase signaling. Via expression of EGFP-tagged SOS in B cells, they revealed that activated and membrane-translocated SOS remains in the membrane for more than $10 \mathrm{~min}$. By half an hour after stimulation, most SOS protein seemed to appear in perinuclear vesicular structures, suggesting that SOS is incapable of dissociating from the membrane, instead being subjected to endocytosis [13] (Fig. 1).

\subsection{Ras inhibition by direct tyrosine phosphorylation}

In the first report on Ras phosphorylation in 1989 [20], the authors reported that purified H-Ras was phosphorylated in vitro by insulin receptor in the presence of poly(L-lysine). Interestingly, poly(L-lysine) was not necessary for K-Ras phosphorylation in the same assay system, perhaps due to the unique, extremely basic hypervariable region of K-Ras, which might effectively substitute for the poly(L-lysine). Abl tyrosine kinase was also reported to phosphorylate $\mathrm{H}$ Ras on tyrosine residue 137 [21]. Tyrosine phosphorylation of H-Ras by Abl allosterically enhanced the binding of H-Ras to its effector protein Raf. Serine/threonine phosphorylation of Ras proteins may also play important roles in their regulation. For example, H-Ras phosphorylation by GSK3 $\beta$ on threonine residues 144 and 148 results in polyubiquitination and proteasome-mediated degradation [22]. Protein kinase Cdependent $\mathrm{K}$-Ras4B phosphorylation was also found on Ser181, which is located in the C-terminal hypervariable polybasic region. Phosphorylation of K-Ras S181 induces rapid translocation of the protein from the plasma membrane to internal membranes, including the endoplasmic reticulum and the mitochondrial membrane. Since phosphorylated KRas interacts with $\mathrm{Bcl}-\mathrm{xL}$ at the outer mitochondrial membrane, K-Ras was implicated in cell death regulation [23, 24]. Recently, a poorly characterized serine/threonine kinase, STK19, was identified as a novel activator of N-Ras [25]. STK19 phosphorylates N-Ras on the evolutionarily conserved Ser89 in the protein's $\alpha 3$-helix. Ser89 phosphorylation facilitates interactions between N-Ras and its downstream effectors, e.g., B-Raf and PI $3 \mathrm{~K} \alpha$, resulting in increased activation of the MAP kinase cascade and the PI3K pathway. The potential therapeutic effect of a STK19 inhibitor was successfully tested in a melanoma model. It was shown that the selective STK19 inhibitor ZT-12-037 (1a) markedly inhibited N-Rasdriven melanoma development and growth both in vitro and in animals [25] (Fig. 2).

The cooperation between Src and Ras in cell growth regulation and tumorigenesis has been known for years. For example, both Ras and Src are downstream members of epidermal growth factor (EGF) signaling $[8,9,26]$. Viral Src can phosphorylate the adaptor protein Shc, which then recruits Grb2/SOS complexes for Ras activation at the plasma membrane [27]. Ras has been shown to be essential for $\mathrm{v}-\mathrm{Src}-$ stimulated cell transformation of human gallbladder epithelial cells or invasive pancreatic ductal adenocarcinoma [28, 29]. p120RasGAP was identified as an effector of c-Src activation by oncogenic Ras [30]. Furthermore, Src cooperates with mutant Ras in tumourigenesis through the JNK and PI3K pathways [31], although no direct relationship between Src and Ras has been identified in this process. Recent studies from the laboratory of Michel Ohh demonstrated that Src tyrosine kinase directly phosphorylates Ras on tyrosine 32 within the 


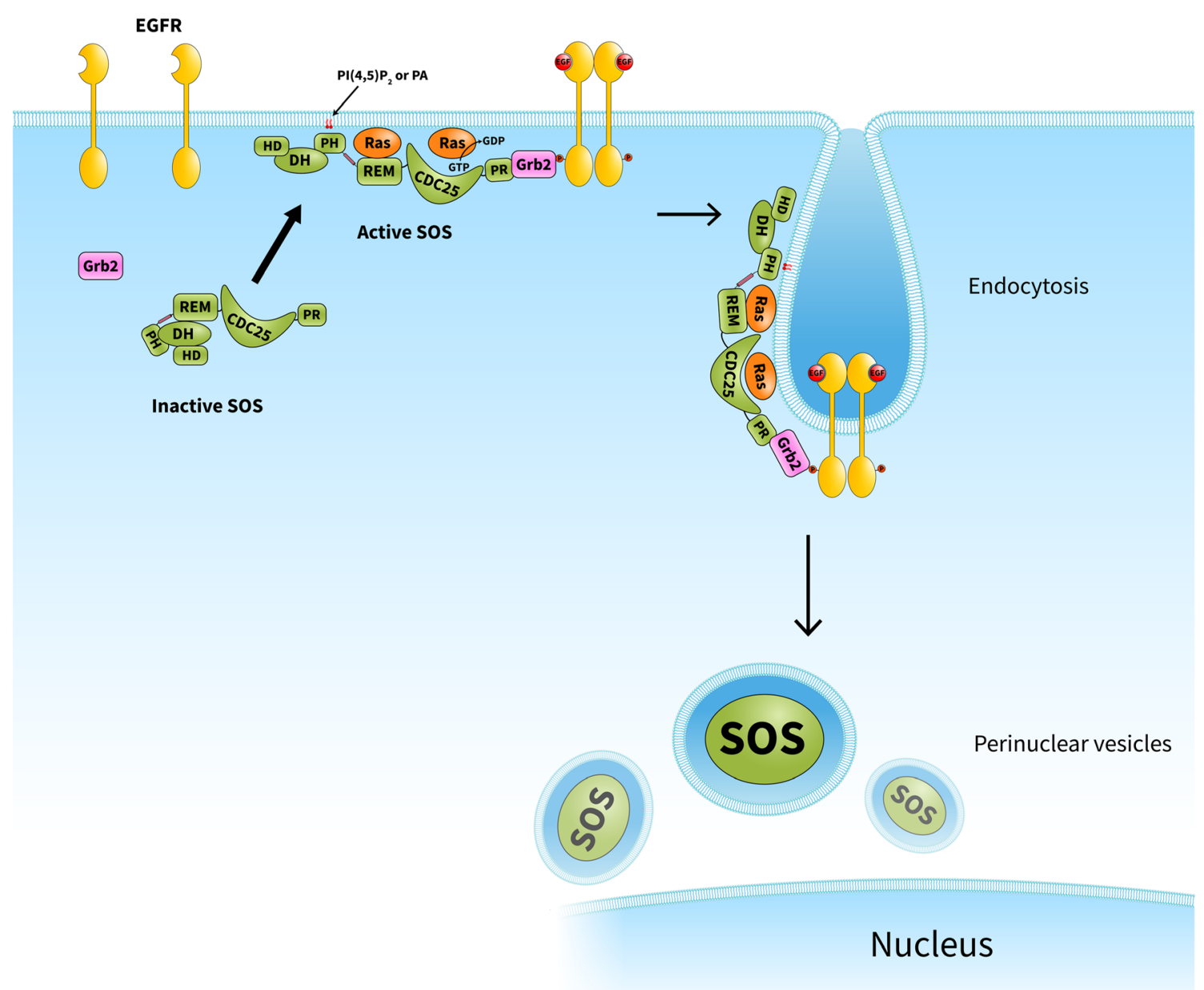

Fig. 1 Model for SOS-dependent Ras activation upon growth factor stimulation. In quiescent cells, SOS is maintained in an inactive conformation by the autoinhibitory function of its PH-DH domains. In response to growth factor treatment, SOS is initially recruited to the membrane via at least two independent sites: a proline-rich Grb2binding site and its lipid-binding PH domain. Contact between the PH domain and phospholipids induces conformational changes that allow allosteric Ras binding, which is followed by processive substrate Ras activation. SOS remains bound at the plasma membrane for minutes and is then subjected to endocytosis. As SOS ultimately seems to appear in perinuclear vesicles, it is likely that SOS translocation to the membrane in response to growth factor stimulation is a one-way process. CDC25, CDC25 homology domain; DH, Dbl homology domain; EGFR, epidermal growth factor receptor; HD, histone-like domain; $\mathrm{PH}$, Pleckstrin homology domain; PA, phosphatidic acid; PR, proline-rich domain; REM, Ras exchanger motif
Fig. 2 Phosphorylation site of Ras proteins. Schematic representation of the phosphorylation sites discussed in this review. GSK $3 \beta$, glycogen synthase kinase $3 \beta$; PKA, protein kinase A; PKC, protein kinase $\mathrm{C}$; STK19, serine/threonine-protein kinase 19
H-Ras

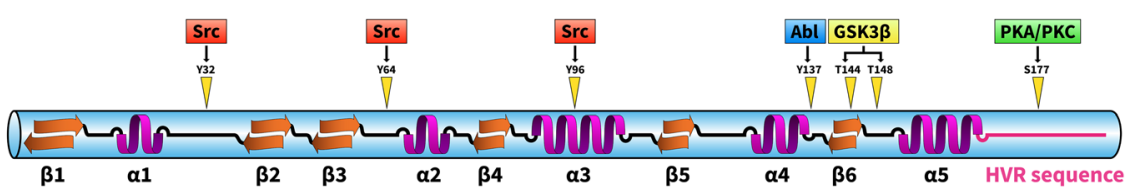

K-Ras

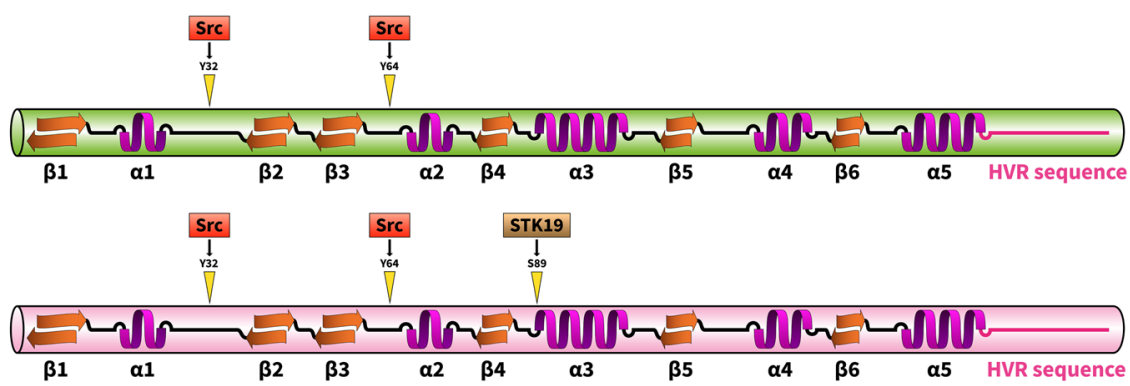


switch I region [32]. Interestingly, Src can bind to and phosphorylate Ras, but only the GTP-bound form; therefore, it is highly likely that Src distinguishes between the different conformations of Ras and can bind only to the activated $\mathrm{G}$ protein. Ras tyrosine 32 phosphorylation has two important effects on Ras signaling: phosphorylation inhibits the binding of the effector protein Raf while increases the binding of the GAP protein leading to enhanced GTP hydrolysis [32]. Both changes facilitate a conformational shift in Ras that inactivates the Ras cycle.

Real-time nuclear magnetic resonance experiments have shown that Src kinase phosphorylates not only Tyr32 in KRas but also Tyr64 [33]. This activity is particularly interesting since Tyr32 is located in the switch I region (aa 30-38) while Tyr64 is positioned in the switch II sequence of K-Ras (aa 59-76). These regions of Ras undergo conformational changes when GDP is exchanged for GTP. In the GTPbound state, Tyr35 and Gly60 form hydrogen bonds with the $\gamma$-phosphate and lock the switch I and II regions in the active conformations [17]. Both the switch I and II regions are implicated in the binding of effectors and GAP proteins. After GTP hydrolysis, the $\gamma$-phosphate is released and both switch regions return to the flexible conformation in the GDP-bound state [17]. Considering the important roles of the switch regions in Ras regulation, it is not surprising that Tyr32 and Tyr64 phosphorylation markedly alters the conformation of the switch I and II regions, negatively influencing every step of the Ras cycle [33]. As such, K-Ras tyrosine phosphorylation significantly reduces the associated GEF and GAP activities and impairs binding of the Raf effector molecule [33].

The crystal structure of nucleotide-free Ras in complex with the catalytic domain of SOS revealed that SOS induces conformational changes in the switch I and II regions of Ras [34]. Mutagenesis of residues at the switch II-SOS interface showed that the primary contact residue is Tyr64, which was buried in a hydrophobic pocket of SOS [35]. Interestingly, the switch I region of Ras is also involved in the interaction with SOS. Mutagenic analysis revealed that Tyr32 and Tyr40 play fundamental roles in this contact. Mutation of these residues resulted in an increase in the intrinsic rate of nucleotide exchange and decreased the binding of Ras to SOS [35]. These findings largely explain why Tyr32 and Tyr64 phosphorylation in Ras, resulting in the incorporation of phosphate groups at the contact sites of Ras and SOS, impairs GEF activity.

\subsection{Ras activation by the SHP2 phosphoprotein phosphatase}

SH2 domain-containing protein tyrosine phosphatase 2 (SHP2), encoded by PTPN11, is associated with a number of malignant conditions, including breast cancer, leukemia, lung cancer, liver cancer, gastric cancer, and other cancer types [36]. Germline-activating mutations in the PTPN11 gene cause Noonan syndrome, whereas somatic mutations result in LEOPARD syndrome and childhood leukemia [36]. The protein phosphatase contains two SH2 domains, a PTP catalytic domain and a C-terminal tail. The crystal structure of the SHP2 tyrosine phosphatase has revealed how its catalytic activity is regulated by its two $\mathrm{SH} 2$ domains. In the absence of a tyrosine-phosphorylated-binding partner, SHP2 is kept in an autoinhibited conformation by intramolecular interactions between the N-terminal SH2 domain and the phosphatase domain. However, in response to growth factor or cytokine stimulation, binding of specific phosphotyrosine proteins to the $\mathrm{N}$ terminal SH2 domain releases the autoinhibition and activates SHP2. Recognition of bisphosphorylated ligands by the tandem SH2 domains is an integral element of the activation. The C-terminal SH2 domain contributes binding energy and defines specificity, but it does not have a direct role in activation $[36,37]$.

In 2007, PTPN11 (the SHP2-encoding gene) was identified as the first proto-oncogene that encodes a tyrosine phosphatase [38]. Although phosphatases are generally thought to be negative regulators of signaling pathways involving protein kinases, several lines of evidence suggest that SHP2 promotes growth factor- and cytokine-induced Ras activation [39]; therefore, SHP2 has long been considered as a potential therapeutic target in cancer treatment. It was demonstrated that SHP2 inhibition blocks signaling from receptor tyrosine kinases (e.g., the EGF receptor) to the MEK-ERK pathway [40]. SHP2 inhibition seemed lethal to cells that are driven by activated tyrosine kinases, and it was implicated in intrinsic and acquired resistance to targeted cancer drugs [40]. Other data demonstrated that pharmacological SHP2 inhibition is an effective therapeutic approach for cancer treatment. A selective and orally bioavailable small-molecule SHP2 inhibitor, SHP099, can block the Ras-Erk signaling pathway activated by mutant receptor tyrosine kinases [41]. Interestingly, SHP099 binding to the interface of the N-terminal SH2 domain and the tyrosine phosphatase domain stabilizes SHP2 in the autoinhibited confirmation [41]. SHP2 inhibition alone had very little or no effect on proliferation in non-small-cell lung cancer (NSCLCs) cell lines; however, a combination of SHP2 and MEK inhibitors showed marked synergy in KRAS-mutant NSCLC cells and in animal models [42, 43]. Finally, studies in which SHP2 was targeted with the small allosteric inhibitor RMC-4550 demonstrated that it can be an effective target in B-Raf mutant cancer cell lines. It was proposed that the mechanism of the inhibitory effect involves disruption of SOS-mediated Ras GTP loading [44].

Although several promising SHP2 inhibitors for use in cancer therapy have been described in the last decade, the precise mechanism by which SHP2 promotes Ras activation remains fundamentally unclear. Bunda et al. identified for the first time that SHP2 preferentially binds to and dephosphorylates PTyr32 Ras [45]. They showed that SHP2 
Fig. 3 Proposed model of the phosphorylation-dependent Ras GTPase cycle. Normal Ras signaling: EGF receptor stimulation leads to simultaneous activation of the SOS exchange factor and Src tyrosine kinase. GTP-bound Ras is a substrate of Src, which phosphorylates Ras on tyrosine 32 and tyrosine 64 , resulting in Ras inactivation. Release from this inhibition can be achieved via the action of SHP2 phosphatase, which dephosphorylates Ras protein. Mutant Ras signaling: oncogenic mutations activate Ras via a defect in its ability to hydrolyze GTP, thus locking Ras in a GTPbound state. Src phosphorylates the constitutively active Ras, thus leading to its inactivation. SHP2 dephosphorylates Ras allowing downstream signaling. Therefore, SHP2 is a novel drug target that might be effective in Ras-driven human cancer

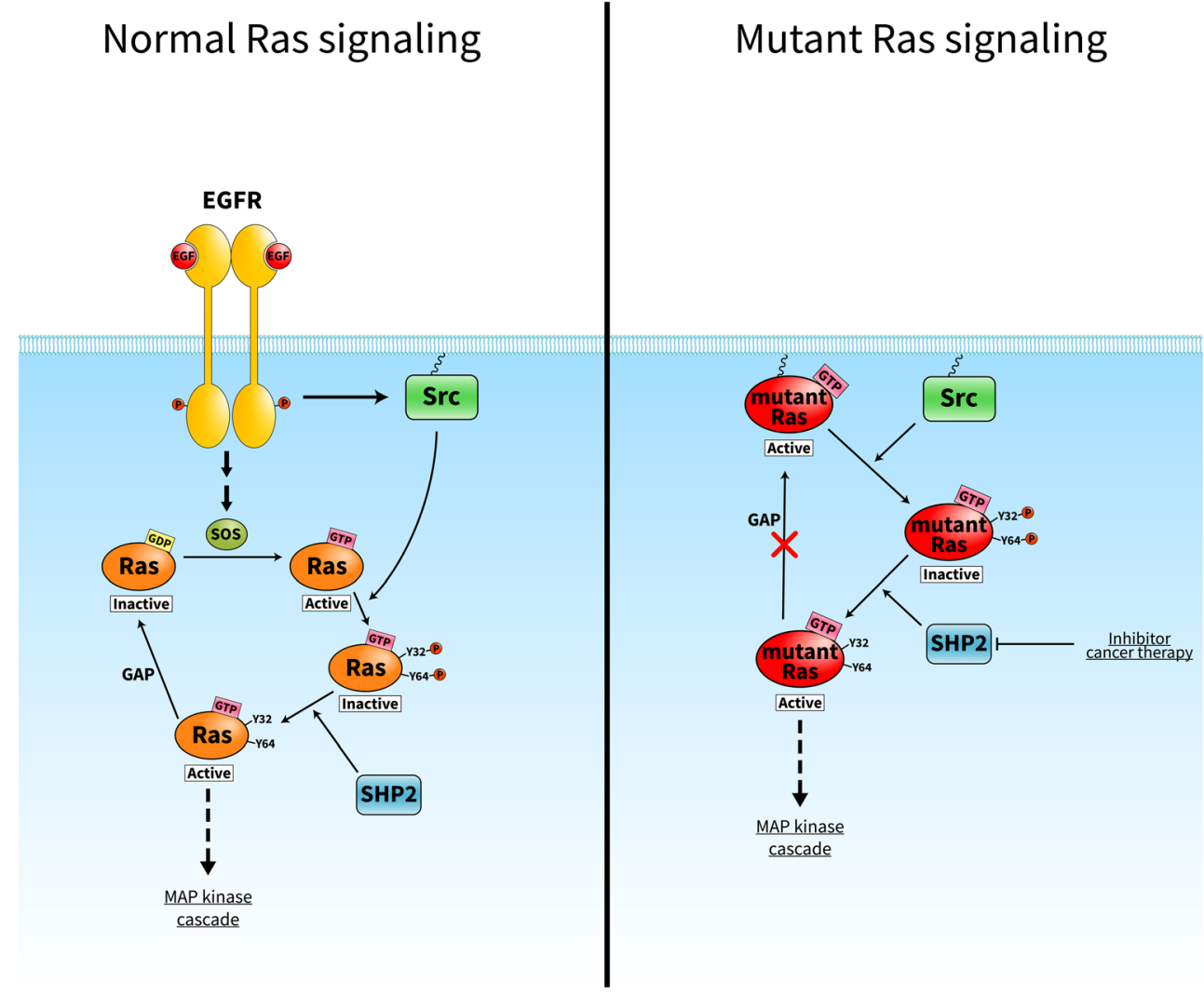

dephosphorylates wild-type and mutant Ras, and the activities of SHP2 and Ras were elevated in mouse and human glioblastoma multiforme cell lines. In addition, SHP2 inhibition facilitated phosphorylation of wild-type and oncogenic Ras, resulting in inactivation of Ras and its downstream signaling [45]. The above findings suggest the existence of another layer of Ras regulation that might fine-tune the classical GEF/ GAP-mediated Ras cycle. In response to, for example, EGF stimulation, Src tyrosine kinase is activated at the plasma membrane where it preferentially phosphorylates GTPbound Ras in which the Tyr32 hydroxyl-group is rotated in the optimal position for phosphorylation [32]. Tyrosine phosphorylation negatively regulates Ras, even in its GTP-bound state, and blocks the Ras cycle. Release from this inhibition can be achieved via the action of SHP2 phosphatase, which dephosphorylates Ras protein (Fig. 3). What is the rationale behind this regulation in terms of cancer treatment? Ras mutations lead to decreased intrinsic Ras GTPase activity and/or impaired binding of GAP protein to Ras, both of which lead to sustained and elevated Ras activation. Mutant GTP-bound Ras acts as a driver that contributes to the pathogenesis of several cancer types. For many decades, Ras was thought to be an "undruggable" cancer target, since no drug against Ras had been approved by authorities [46]. The discovery that SHP2 can dephosphorylate even mutant GTP-bound Ras in tumor cells and release Ras from its inactive state establishes
SHP2 as a novel drug target that could be effective in Rasdriven human cancer.

Acknowledgments We are grateful to Tamás Takács for drawing the figures.

Funding information Open access funding provided by ELKH Research Centre for Natural Sciences. This research was funded by grants from the National Research, Development, and Innovation Fund of Hungary (K 124045, FIEK 16-1-2016-0005 and HunProEx 2018-1.2.1-NKP-201800005); the MedinProt Program of the Hungarian Academy of Sciences (LB). The work of Virag Vas was supported by a János Bolyai Research Scholarship from the Hungarian Academy of Sciences.

\section{Compliance with ethical standards}

Conflict of interests The authors declare that they have no conflicts of interest.

Open Access This article is licensed under a Creative Commons Attribution 4.0 International License, which permits use, sharing, adaptation, distribution and reproduction in any medium or format, as long as you give appropriate credit to the original author(s) and the source, provide a link to the Creative Commons licence, and indicate if changes were made. The images or other third party material in this article are included in the article's Creative Commons licence, unless indicated otherwise in a credit line to the material. If material is not included in the article's Creative Commons licence and your intended use is not permitted by statutory regulation or exceeds the permitted use, you will need to obtain 
permission directly from the copyright holder. To view a copy of this licence, visit http://creativecommons.org/licenses/by/4.0/.

\section{References}

1. Lowy, D. R., \& Willumsen, B. M. (1993). Function and regulation of ras. Annual Review of Biochemistry, 62, 851-891.

2. Hobbs, G. A., Der, C. J., \& Rossman, K. L. (2016). RAS isoforms and mutations in cancer at a glance. Journal of Cell Science, 129, $1287-1292$.

3. Downward, J. (1996). Control of ras activation. Cancer Surveys, $27,87-100$.

4. Bos, J. L., Rehmann, H., \& Wittinghofer, A. (2007). GEFs and GAPs: critical elements in the control of small $G$ proteins. Cell., 129, 865-877.

5. Miller, M. S., \& Miller, L. D. (2011). RAS mutations and oncogenesis: not all RAS mutations are created equally. Frontiers in Genetics, 2, 100.

6. Prior, I. A., Lewis, P. D., \& Mattos, C. (2012). A comprehensive survey of Ras mutations in cancer. Cancer Research, 72, 2457 2467.

7. Forbes, S. A., Bindal, N., Bamford, S., Cole, C., Kok, C. Y., Beare, D., Jia, M., Shepherd, R., Leung, K., Menzies, A., Teague, J. W., Campbell, P. J., Stratton, M. R., \& Futreal, P. A. (2011). COSMIC: mining complete cancer genomes in the catalogue of somatic mutations in cancer. Nucleic Acids Research, 39, D945-D950.

8. Egan, S. E., Giddings, B. W., Brooks, M. W., Buday, L., Sizeland, A. M., \& Weinberg, R. A. (1993). Association of Sos Ras exchange protein with Grb2 is implicated in tyrosine kinase signal transduction and transformation. Nature., 363, 45-51.

9. Buday, L., \& Downward, J. (1993). Epidermal growth factor regulates p21ras through the formation of a complex of receptor, Grb2 adapter protein, and Sos nucleotide exchange factor. Cell., 73, 611620.

10. Buday, L., \& Downward, J. (2008). Many faces of Ras activation. Biochimica et Biophysica Acta, 1786, 178-187.

11. Wang, W., Fisher, E. M., Jia, Q., Dunn, J. M., Porfiri, E., Downward, J., \& Egan, S. E. (1995). The Grb2 binding domain of mSos1 is not required for downstream signal transduction. Nature Genetics, 10, 294-300.

12. Zarich, N., Oliva, J. L., Martinez, N., Jorge, R., Ballester, A., Gutierrez-Eisman, S., Garcia-Vargas, S., \& Rojas, J. M. (2006). Grb2 is a negative modulator of the intrinsic Ras-GEF activity of hSos1. Molecular Biology of the Cell, 17, 3591-3597.

13. Christensen, S. M., Tu, H. L., Jun, J. E., Alvarez, S., Triplet, M. G., Iwig, J. S., Yadav, K. K., Bar-Sagi, D., Roose, J. P., \& Groves, J. T. (2016). One-way membrane trafficking of SOS in receptortriggered Ras activation. Nature Structural \& Molecular Biology, $23,838-846$.

14. Sondermann, H., Soisson, S. M., Boykevisch, S., Yang, S. S., BarSagi, D., \& Kuriyan, J. (2004). Structural analysis of autoinhibition in the Ras activator son of sevenless. Cell., 119, 393-405.

15. Lee, Y. K., Low-Nam, S. T., Chung, J. K., Hansen, S. D., Lam, H. Y. M., Alvarez, S., \& Groves, J. T. (2017). Mechanism of SOS PRdomain autoinhibition revealed by single-molecule assays on native protein from lysate. Nature Communications, $8,15061$.

16. Findlay, G. M., \& Pawson, T. (2008). How is SOS activated? Let us count the ways. Nature Structural \& Molecular Biology, 15, 538540 .

17. Simanshu, D. K., Nissley, D. V., \& McCormick, F. (2017). RAS proteins and their regulators in human disease. Cell., 170, 17-33.
18. Aronheim, A., Engelberg, D., Li, N., Al-Alawi, N., Schlessinger, J., \& Karin, M. (1994). Membrane targeting of the nucleotide exchange factor Sos is sufficient for activating the Ras signaling pathway. Cell., 78, 949-961.

19. Iversen, L., Tu, H. L., Lin, W. C., Christensen, S. M., Abel, S. M., Iwig, J., Wu, H. J., Gureasko, J., Rhodes, C., Petit, R. S., Hansen, S. D., Thill, P., Yu, C. H., Stamou, D., Chakraborty, A. K., Kuriyan, J., \& Groves, J. T. (2014). Molecular kinetics. Ras activation by SOS: allosteric regulation by altered fluctuation dynamics. Science., 345, 50-54.

20. Fujita-Yamaguchi, Y., Kathuria, S., Xu, Q. Y., McDonald, J. M., Nakano, H., \& Kamata, T. (1989). In vitro tyrosine phosphorylation studies on RAS proteins and calmodulin suggest that polylysinelike basic peptides or domains may be involved in interactions between insulin receptor kinase and its substrate. Proceedings of the National Academy of Sciences of the United States of America, 86, 7306-7310.

21. Ting, P. Y., Johnson, C. W., Fang, C., Cao, X., Graeber, T. G., Mattos, C., \& Colicelli, J. (2015). Tyrosine phosphorylation of RAS by ABL allosterically enhances effector binding. The FASEB Journal, 29, 3750-3761.

22. Jeong, W. J., Yoon, J., Park, J. C., Lee, S. H., Lee, S. H., Kaduwal, S., Kim, H., Yoon, J. B., \& Choi, K. Y. (2012). Ras stabilization through aberrant activation of Wnt/beta-catenin signaling promotes intestinal tumorigenesis. Science Signaling, 5, ra30.

23. Bivona, T. G., Quatela, S. E., Bodemann, B. O., Ahearn, I. M., Soskis, M. J., Mor, A., Miura, J., Wiener, H. H., Wright, L., Saba, S. G., Yim, D., Fein, A., Perez de Castro, I., Li, C., Thompson, C. B., Cox, A. D., \& Philips, M. R. (2006). PKC regulates a farnesyl-electrostatic switch on K-Ras that promotes its association with Bcl-XL on mitochondria and induces apoptosis. Molecular Cell, 21, 481-493.

24. Sung, P. J., Tsai, F. D., Vais, H., Court, H., Yang, J., Fehrenbacher, N., Foskett, J. K., \& Philips, M. R. (2013). Phosphorylated K-Ras limits cell survival by blocking Bcl-xL sensitization of inositol trisphosphate receptors. Proceedings of the National Academy of Sciences of the United States of America, 110, 20593-20598.

25. Yin, C., Zhu, B., Zhang, T., Liu, T., Chen, S., Liu, Y., Li, X., Miao, X., Li, S., Mi, X., Zhang, J., Li, L., Wei, G., Xu, Z. X., Gao, X., Huang, C., Wei, Z., Goding, C. R., Wang, P., Deng, X., \& Cui, R. (2019). Pharmacological targeting of STK19 inhibits oncogenic NRAS-driven melanomagenesis. Cell., 176(1113-1127), e16.

26. Belsches, A. P., Haskell, M. D., \& Parsons, S. J. (1997). Role of cSrc tyrosine kinase in EGF-induced mitogenesis. Frontiers in Bioscience, 2, d501-d518.

27. van der Geer, P., Wiley, S., Gish, G. D., \& Pawson, T. (1996). The Shc adaptor protein is highly phosphorylated at conserved, twin tyrosine residues (Y239/240) that mediate protein-protein interactions. Current Biology, 6, 1435-1444.

28. Tokumitsu, Y., Nakano, S., Ueno, H., \& Niho, Y. (2000). Suppression of malignant growth potentials of v-Src-transformed human gallbladder epithelial cells by adenovirus-mediated dominant negative H-Ras. Journal of Cellular Physiology, 183, 221227.

29. Shields, D. J., Murphy, E. A., Desgrosellier, J. S., Mielgo, A., Lau, S. K., Barnes, L. A., Lesperance, J., Huang, M., Schmedt, C., Tarin, D., Lowy, A. M., \& Cheresh, D. A. (2011). Oncogenic Ras/Src cooperativity in pancreatic neoplasia. Oncogene., 30, 2123-2134.

30. Chan, P. C., \& Chen, H. C. (2012). p120RasGAP-mediated activation of c-Src is critical for oncogenic Ras to induce tumor invasion. Cancer Research, 72, 2405-2415.

31. Poon, C. L. C., Brumby, A. M., \& Richardson, H. E. (2018). Src cooperates with oncogenic Ras in tumourigenesis via the JNK and PI3K pathways in drosophila epithelial tissue. International Journal of Molecular Sciences, 19, 1585. 
32. Bunda, S., Heir, P., Srikumar, T., Cook, J. D., Burrell, K., Kano, Y., Lee, J. E., Zadeh, G., Raught, B., \& Ohh, M. (2014). Src promotes GTPase activity of Ras via tyrosine 32 phosphorylation. Proceedings of the National Academy of Sciences of the United States of America, 111, E3785-E3794.

33. Kano, Y., Gebregiworgis, T., Marshall, C. B., Radulovich, N., Poon, B. P. K., St-Germain, J., Cook, J. D., Valencia-Sama, I., Grant, B. M. M., Herrera, S. G., Miao, J., Raught, B., Irwin, M. S., Lee, J. E., Yeh, J. J., Zhang, Z. Y., Tsao, M. S., Ikura, M., \& Ohh, M. (2019). Tyrosyl phosphorylation of KRAS stalls GTPase cycle via alteration of switch I and II conformation. Nature Communications, 10, 224.

34. Boriack-Sjodin, P. A., Margarit, S. M., Bar-Sagi, D., \& Kuriyan, J. (1998). The structural basis of the activation of Ras by Sos. Nature., 394, 337-343.

35. Hall, B. E., Yang, S. S., Boriack-Sjodin, P. A., Kuriyan, J., \& BarSagi, D. (2001). Structure-based mutagenesis reveals distinct functions for Ras switch 1 and switch 2 in Sos-catalyzed guanine nucleotide exchange. The Journal of Biological Chemistry, 276, 27629-27637.

36. Frankson, R., Yu, Z. H., Bai, Y., Li, Q., Zhang, R. Y., \& Zhang, Z. $Y$. (2017). Therapeutic targeting of oncogenic tyrosine phosphatases. Cancer Research, 77, 5701-5705.

37. Hof, P., Pluskey, S., Dhe-Paganon, S., Eck, M. J., \& Shoelson, S. E. (1998). Crystal structure of the tyrosine phosphatase SHP-2. Cell., 92, 441-450.

38. Chan, R. J., \& Feng, G. S. (2007). PTPN11 is the first identified proto-oncogene that encodes a tyrosine phosphatase. Blood., 109, 862-867.

39. Matozaki, T., Murata, Y., Saito, Y., Okazawa, H., \& Ohnishi, H. (2009). Protein tyrosine phosphatase SHP-2: a proto-oncogene product that promotes Ras activation. Cancer Science, 100, 17861793.

40. Prahallad, A., Heynen, G. J., Germano, G., Willems, S. M., Evers, B., Vecchione, L., Gambino, V., Lieftink, C., Beijersbergen, R. L., Di Nicolantonio, F., Bardelli, A., \& Bernards, R. (2015). PTPN11 is a central node in intrinsic and acquired resistance to targeted cancer drugs. Cell Reports, 12, 1978-1985.

41. Chen, Y. N., LaMarche, M. J., Chan, H. M., Fekkes, P., GarciaFortanet, J., Acker, M. G., Antonakos, B., Chen, C. H., Chen, Z., Cooke, V. G., Dobson, J. R., Deng, Z., Fei, F., Firestone, B., Fodor, M., Fridrich, C., Gao, H., Grunenfelder, D., Hao, H. X., Jacob, J.,
Ho, S., Hsiao, K., Kang, Z. B., Karki, R., Kato, M., Larrow, J., La Bonte, L. R., Lenoir, F., Liu, G., Liu, S., Majumdar, D., Meyer, M. J., Palermo, M., Perez, L., Pu, M., Price, E., Quinn, C., Shakya, S., Shultz, M. D., Slisz, J., Venkatesan, K., Wang, P., Warmuth, M., Williams, S., Yang, G., Yuan, J., Zhang, J. H., Zhu, P., Ramsey, T., Keen, N. J., Sellers, W. R., Stams, T., \& Fortin, P. D. (2016). Allosteric inhibition of SHP2 phosphatase inhibits cancers driven by receptor tyrosine kinases. Nature., 535, 148-152.

42. Mainardi, S., Mulero-Sanchez, A., Prahallad, A., Germano, G., Bosma, A., Krimpenfort, P., Lieftink, C., Steinberg, J. D., de Wit, N., Goncalves-Ribeiro, S., Nadal, E., Bardelli, A., Villanueva, A., \& Bernards, R. (2018). SHP2 is required for growth of KRASmutant non-small-cell lung cancer in vivo. Nature Medicine, 24, 961-967.

43. Ruess, D. A., Heynen, G. J., Ciecielski, K. J., Ai, J., Berninger, A., Kabacaoglu, D., Gorgulu, K., Dantes, Z., Wormann, S. M., Diakopoulos, K. N., Karpathaki, A. F., Kowalska, M., KayaAksoy, E., Song, L., van der Laan, E. A. Z., Lopez-Alberca, M. P., Nazare, M., Reichert, M., Saur, D., Erkan, M. M., Hopt, U. T., Sainz Jr., B., Birchmeier, W., Schmid, R. M., Lesina, M., \& Algul, H. (2018). Mutant KRAS-driven cancers depend on PTPN11/SHP2 phosphatase. Nature Medicine, 24, 954-960.

44. Nichols, R. J., Haderk, F., Stahlhut, C., Schulze, C. J., Hemmati, G., Wildes, D., Tzitzilonis, C., Mordec, K., Marquez, A., Romero, J., Hsieh, T., Zaman, A., Olivas, V., McCoach, C., Blakely, C. M., Wang, Z., Kiss, G., Koltun, E. S., Gill, A. L., Singh, M., Goldsmith, M. A., Smith, J. A. M., \& Bivona, T. G. (2018). RAS nucleotide cycling underlies the SHP2 phosphatase dependence of mutant BRAF-, NF1- and RAS-driven cancers. Nature Cell Biology, 20, 1064-1073.

45. Bunda, S., Burrell, K., Heir, P., Zeng, L., Alamsahebpour, A., Kano, Y., Raught, B., Zhang, Z. Y., Zadeh, G., \& Ohh, M. (2015). Inhibition of SHP2-mediated dephosphorylation of Ras suppresses oncogenesis. Nature Communications, 6, 8859.

46. Stalnecker, C. A., \& Der, C. J. (2020). RAS, wanted dead or alive: advances in targeting RAS mutant cancers. Science Signaling, 13, 624.

Publisher's note Springer Nature remains neutral with regard to jurisdictional claims in published maps and institutional affiliations. 